\title{
Neurotherapeutics of the Aging Brain: Complexity Meets Complexity
}

\author{
Alan J. Lerner ${ }^{1,2}$ (1) Andrew A. Pieper ${ }^{3,4,5}$ \\ Published online: 9 July 2019 \\ (C) The American Society for Experimental NeuroTherapeutics, Inc. 2019
}

Both "aging" and the "brain" are vast topics that certainly merit study each in their own right, but when placed together, one seems hard put to know where to start. For many years, it has been fashionable to pose questions to trainees and colleagues such as "Why do we age?" as if a question so simple could easily yield profound insights. One's first temptation is to conclude that time passes and therefore things get older. However, this ignores the fact that aging is a process that appears to result from the confluence of genetic and environmental factors. Indeed, one definition of aging is that of a coordinated response to a changing environment, both internal and external, that culminates in magnitude as we approach the end of the life span. In that many age-associated changes in our bodies are associated with increased susceptibility to injury and disease, there is merit in exploring how to mitigate the effects of aging in order to lead healthier, happier, and longer lives.

As with the general phenomenon of aging, the brain is equally inscrutable in revealing its secrets and properties. Whether the human brain is the most complex "thing" is debatable, but nobody seems to doubt that the process of the brain understanding itself is truly remarkable, almost science fiction in its improbability, yet real and palpable with regular

Alan J. Lerner

Alan.Lerner@uhhospitals.org

Andrew A. Pieper

Andrew.Pieper@harringtondiscovery.org

1 Department of Neurology, University Hospitals Cleveland Medical Center, Cleveland, $\mathrm{OH}$, USA

2 Department of Neurology, Case Western Reserve University, 3619 Park east Drive, suite 206, Beachwood, OH 44122, USA

3 Department of Psychiatry, Case Western Reserve University, Cleveland, OH, USA

4 Harrington Discovery Institute, University Hospitals Cleveland Medical Center, Cleveland, OH, USA

5 Geriatric Research and Education and Clinical Center, Louis Stokes Cleveland VAMC, Cleveland, OH 44106, USA advances improving the human condition. Given the rapidly accelerating aging of populations throughout the world - in the USA, Europe, Japan, and China - the ability to intervene in meaningful ways across our advancing years has become a top scientific and medical priority.

In this special issue of Neurotherapeutics, a wide range of articles bring the reader into the latest developments in the field of healthy brain aging. The challenge in the field is to create a coherent narrative of what appear to be isolated strands, and combine multiple physiologic changes associated with myriad disease states into efficient and doable therapeutics over wide populations. Several papers in this issue approach these weighty issues from a wide-ranging perspective, while others explore a single system or therapeutic modality in greater detail.

To begin, Lupo et al. [1] review molecular signatures of the aging brain. This approach of utilizing basic science as a platform to devise interventions is still in its early stages of application to the aging brain. Which of the many -omics approaches will ultimately lead to specific interventions to improve brain health remains unsettled, but cooperative science across large groups of investigators is accelerating the discovery of knowledge and guiding future interventions. The breadth of any individual approach will need to be considered, since multiple feedback loops and buffering of genomic, proteomic, and metabolomic systems underscore the significant challenge in finding a "magic bullet" within the tangles of these systems.

Next, Bah et al. [2] review sleep as a therapeutic target for healthy brain aging. Maintaining proper sleep is a major clinical challenge in neurodegenerative diseases of aging that are characterized by prominent sleep disturbances, such as Parkinson's disease (PD), Dementia with Lewy bodies, variants of prion disorders, and Alzheimer's disease (AD). Further, sleep deprivation is endemic in our society, and in some demanding professions, the extent of sleep deprivation endured by individuals striving for productivity in their field is paradoxically worn as a badge of honor. The long-term negative health effects of chronic sleep deprivation and disturbance are profound, and this remains an active area of research. For 
example, sleep, or more accurately the sleep-wake cycle, is associated with cellular repair processes as well as clearance of amyloid and other interstitial waste products through the glymphatic system, a critical component of maintaining healthy brain physiology [3]. The obvious nonpharmacologic approach of fostering adherence to healthy sleep habits is particularly challenging as it requires consistent behavioral changes across the life span that become even more challenging as health conditions associated with aging emerge, such as neurodegenerative disease. While melatonin can be effectively used to stabilize the sleep-wake cycle in normal physiology, translation into concrete benefit in neurodegenerative disorders associated with aging has been minimal to date. Furthermore, careful attention needs to be devoted to minimizing the adverse effects of sedative-hypnotic sleep aids, as historically this area has been fraught with complications, such as the well-known adverse effects of gamma-hydroxybutyrate that have limited its usage in clinical practice. Newer therapeutic modalities are thus currently in development to promote healthy sleep in the aging brain, such as GABA transporter 1 inhibitors, GABA-A agonists, and GABA-B modulators. Modulation of other systems, such as the serotonin receptor, are also in development [4].

Beyond maintaining healthy sleep, risk factor reduction for neurodegenerative disorders is further addressed in the next two articles, authored by Wahidi and Lerner [5] and Cabral et al. [6]. Risk factor reduction holds the promise of preventing as much as $25 \%$ of dementias going forward, which would clearly result in a meaningful reduction of societal and individual burden. First, Wahidi and Lerner review the effects of hypertension on the brain. The recent SPRINT study [7] has greatly increased interest in risk factor reduction and represents one of the first prospective studies showing significant reduction in mild cognitive impairment (MCI). However, hypertension and its treatment also interact with beta-amyloid and tau in complex ways that are not fully understood, and the effect of blood pressure reduction on $\mathrm{AD}$ or other neurodegenerative disorders of aging beyond vascular cognitive impairment is unknown. Cabral et al. [6] next address the effects of exercise on brain health, defined widely. Importantly, exercise can be reconceptualized as a medicine, with both dose effects and risk of adverse outcomes. The content of exercise is also heterogeneous and includes tai chi, aerobic exercise, and many other variants. Physical health benefits of exercise include broad body system effects, such as strength, balance, flexibility, and endurance. Some of these facets can be maximized to support healthy aging of the brain, such as improving balance in people with frequent falls that are common in the later stages of akinetic-rigid syndromes. Exercise is also beneficial for cerebral perfusion, synaptic plasticity, neural network connectivity, and brain structure. Unfortunately, translation into human disease models remains difficult, and disease-specific exercise regimens have yet to reach maturity in clinical translation. However, one notable exception is Parkinson's disease, in which patients respond particularly well to exercise, and a number of studies have also shown promise for exercise in mild cognitive impairment (MCI), itself frequently prodromal of AD $[8,9]$. Given the chronic and progressive aspects of neurodegenerative disorders, exercise can be considered as a long-term intervention with positive effects in many body systems affected by aging, including the brain.

Next, Soon et al. [10] discuss metabolic disturbances that have been widely reported in neurodegenerative disorders associated with aging, especially Alzheimer's disease. These potential targets are interesting not least because our knowledge of metabolic pathways and interactions is extensive, and diabetes mellitus (DM) is a major epidemiological risk factor for $\mathrm{AD}$, as well as a treatable disease amenable to dementia risk factor reduction. Prospective studies of DM treatments applied to $\mathrm{AD}$ are in the early stages and unfortunately have yet to show sustained effects. The authors also highlight how disturbances in circadian rhythm significantly impair overall metabolic balance, which relates to the ideas presented by Bah et al. [2].

We next turn to the role of the lysosome in health and disease, as changes in lysosomal function are present in several neurodegenerative disorders. Here, Peng et al. [11] review the lysosomal system in the aging brain. Linkage of lysosomal function to autophagy has led to studies of mTOR inhibitors such as rapamycin, and the mTOR-dependent stimulator MSDC-0160, in PD, as well as studies of nilotinib in PD and AD. Furthermore, mTOR-independent activators of autophagy are already in clinical practice (e.g., lithium and valproate). Resveratrol, a natural product found in grapes, increased plasma beta-amyloid 1-40, an early indication that $\mathrm{AD}$ biomarkers can be modified in a randomized clinical trial [12]. That autophagy can be affected by many currently available pharmacologic agents, such as metformin and the others mentioned above, is a hopeful sign. Specific interventions in accurately diagnosed patients with known molecular phenotypes will be key to the success of future randomized clinical trials targeted at optimizing lysosomal function in the aging brain.

Another metabolic pathway relevant to the aging brain, reviewed by Petrov and Pikuleva [13], involves cholesterol transport across the blood-brain barrier by CYP46A and its role in AD related to APO E function and amyloid deposition. Previous studies have shown disease-related changes in the functioning of this regulatory system, and 24-hydroxycholesterol (24HC) also interacts with NMDA receptors, which mediate cell injury through excitotoxic neurotransmission. Both genetic and pharmacological modulation of CYP46A1 are under investigation in $\mathrm{AD}$ at present. This includes a novel study of efavirenz, an FDA-approved compound used for HIV disease that also 
shows a favorable profile in modulating CYP46A1 at low doses relative to those used to treat HIV.

Next, Russell et al. [14] report on estrogens in the brain and their effect on cognitive aging. Declining estradiol levels with aging have multiple cognitive and behavioral effects, including impairment in sleep, memory, attention, and mood, and correlative behavioral changes have been well-studied in preclinical models. However, human studies, both longitudinal observational and interventional studies, such as the Women's Health Initiative memory study, have provided conflicting evidence [15]. Further, changes in brain volumes do not necessarily correlate with diminished cognitive outcome. Enthusiasm for this approach also diminished when long-term treatment in the Women's Health Initiative with conjugated equine estrogens with or without medroxyprogesterone led to increased risk of MCI or dementia [16]. The outcomes of further studies with related compounds, such as selective estrogen receptor modulators (SERMs), remain to be seen. In addition, the need to develop interventions for males, and selection of optimal timing for interventions, suggest that precision medicine may yet revive clinical interest in this steroidbased approach to neurodegenerative disorders.

Also in this issue, Singh et al. [17] revisit the potential for Ginkgo biloba as both a neuroprotective agent and antioxidant. Ginkgo also has profound effects on metabolic pathways, including monoamine oxidase inhibition, which may relate to its putative effects as an antidepressant. The GEM study by Dekosky et al. [18] has influenced further research into Ginkgo, in which it unfortunately did not show efficacy in dementia prevention. A similar study showed no evidence of clinical effect in mild to moderate dementia [19], but metabolic parameters moved in the expected direction given the known pharmacology of Ginkgo extracts. Clinicians are aware that Ginkgo is a natural product readily available over the counter and frequently used by patients on their own. All of these facts influence the reluctance of pharmaceutical companies to further investigate these compounds, even as it is widely used on an empirical basis.

Finally, Kheifets and Braithwaite [20] explore a novel approach to therapeutics of the aging brain based on the science of plasma. Much of the work has proceeded in models of normal aging, but early studies in disease models, especially $\mathrm{AD}$, have also been started. The molecular basis of the reported effects, both positive and negative, has led to attempts at identification of "chronokines" using mainly proteomic approaches. Some of these proteins are well-known, such as beta2-microglobulin, whereas others seem surprising such as osteocalcin [21]. Practical problems to be solved include the precautions involved in transfusion of blood products, although further characterization and isolation of proteins capable of positive effects on cognitive function will determine whether this can become a mainstream treatment for $\mathrm{AD}$ or other negative aging-related changes in the brain.
Acknowledgments Alan J. Lerner is supported in part by NIH grants 5R01-AG046543-03, RF1-AG058066, P30-NR015326, 1U24AG057437-01, 5R01 AG053798-02, and NIH contract HHSN26820090049C, and grants from the Alzheimer's Drug Discovery Foundation, Global Alzheimer's Platform, the AHA/Allen Initiative in Brain Health and Cognitive Impairment (19PABH134580006), and the Neurological Institute chair in memory and cognition. Andrew A. Pieper is supported by the Brockman Medical Research Foundation, the AHA/ Allen Initiative in Brain Health and Cognitive Impairment (19PABH134580006), the Elizabeth Ring Mather \& William Gwinn Mather Fund, the S. Livingston Samuel Mather Trust, and the Department of Veterans Affairs Merit Review 1IO1BX002444. The contents of this manuscript do not represent the views of the U.S. Department of Veterans Affairs or the US government.

Required Author Forms Disclosure forms provided by the authors are available with the online version of this article.

\section{References}

1. Lupo G, Gaetani S, Cacci E, Biagioni S, Negri R. Molecular Signatures of the Aging Brain: Finding the Links Between Genes and Phenotypes. Neurotherapeutics, Vol. 16, Number 3, July 2019.

2. Bah TM, Goodman J, Iliff J. Sleep as a therapeutic target in the aging brain. Neurotherapeutics Vol. 16, Number 3, July 2019.

3. Arighi A, Di Cristofori A, Fenoglio C, Borsa S, D'Anca M, Fumagalli GG, Locatelli M, Carrabba G, Pietroboni AM, Ghezzi L, Carandini T. Cerebrospinal Fluid Level of Aquaporin4: A New Window on Glymphatic System Involvement in Neurodegenerative Disease? Journal of Alzheimer's Disease. 2019 May 25(Preprint):1-7.

4. Monti JM, Jantos H. The roles of dopamine and serotonin, and of their receptors, in regulating sleep and waking. Progress in brain research. 2008 Jan 1;172:625-46.

5. Wahidi N, Lerner AJ. Blood Pressure Control and Protection of the Aging Brain. Neurotherapeutics. Vol. 16, Number 3, July 2019.

6. Cabral DF, Rice J, Morris TP, Rundek T, Pascual-Leone A, Gomes Osman Jr. Exercise for brain health: an investigation into the underlying mechanisms guided by dose. Neurotherapeutics. Vol. 16, Number 3, July 2019

7. Williamson JD, Pajewski NM, Auchus AP, Bryan RN, Chelune G, Cheung AK, Cleveland ML, Coker LH, Crowe MG, Cushman WC, Cutler JA. Effect of intensive vs standard blood pressure control on probable dementia: a randomized clinical trial. JAMA. 2019 Feb 12;321(6):553-61

8. Kim Y, Lai B, Mehta T, Thirumalai M, Padalabalanarayanan S, Rimmer JH, Motl RW. Exercise training guidelines for multiple sclerosis, stroke, and Parkinson's disease: Rapid review and synthesis. American Journal of Physical Medicine \& Rehabilitation. March 2019.

9. Loprinzi PD, Blough J, Ryu S, Kang M. Experimental effects of exercise on memory function among mild cognitive impairment: systematic review and meta-analysis. The Physician and Sports Medicine. 2019 Jan 2;47(1):21-6.

10. Yoon SO, Ryu JC, Zimmer ER, Roisa-Neto P. Metabolic disruption in Alzheimer's disease. Neurotherapeutics Vol. 16, Number 3, July 2019

11. Peng W, Minakaki G, Nguyen M, Krainc D. Preserving lysosomal function in the aging brain: Insights from neurodegeneration. Neurotherapeutics Vol. 16, Number 3, July 2019

12. Turner RS, Thomas RG, Craft S, van Dyck CH, Mintzer J, Reynolds BA, Brewer JB, Rissman RA, Raman R, Aisen PS; Alzheimer's Disease Cooperative Study. A randomized, double- 
blind, placebo-controlled trial of resveratrol for Alzheimer disease. Neurology. 2015 Oct 20;85(16):1383-91.

13. Petrov AM, Pikuleva IA. Cholesterol 24-Hydroxylation by CYP46A1: Benefits of Modulation for Brain Diseases. Neurotherapeutics. Vol. 16, Number 3, July 2019

14. Russell JK, Jones CK, Newhouse PA. The role of estrogens in brain and cognitive aging. Neurotherapeutics Vol. 16, Number 3, July 2019

15. Resnick SM1, Espeland MA, Jaramillo SA, Hirsch C, Stefanick ML, Murray AM, Ockene J, Davatzikos C. Postmenopausal hormone therapy and regional brain volumes: the WHIMS-MRI Study. Neurology 2009 Jan 13;72(2):135-42.

16. Petkus AJ, Resnick SM, Rapp SR, Espeland MA, Gatz M, Widaman KF, Wang X, Younan D, Casanova R, Chui H, Barnard RT. General and domain-specific cognitive reserve, mild cognitive impairment, and dementia risk in older women. Alzheimer's \& Dementia: Translational Research \& Clinical Interventions. 2019 Jan 1;5:118-28.

17. Singh SK, Srivastev S, Castellani RJ, Plascencia-Villa G, Perry G, Neuroprotective and antioxidant effect of Ginkgo biloba extract against $\mathrm{AD}$ and other neurological disorders. Neurotherapeutics Vol. 16, Number 3, July 2019
18. DeKosky ST, Williamson JD, Fitzpatrick AL, Kronmal RA, Ives DG, Saxton JA, Lopez OL, Burke G, Carlson MC, Fried LP, Kuller LH. Ginkgo biloba for prevention of dementia: a randomized controlled trial. JAMA. 2008 Nov 19;300(19):2253-62.

19. McCarney R, Fisher P, Iliffe S, van Haselen R, Griffin M, van der Meulen J, Warner J. Ginkgo biloba for mild to moderate dementia in a community setting: a pragmatic, randomised, parallel-group, double-blind, placebo-controlled trial. International Journal of Geriatric Psychiatry: A journal of the psychiatry of late life and allied sciences. 2008 Dec;23(12):1222-30.

20. Kheifets V, Braithwaite SP.Plasma-Based Strategies for Therapeutic Modulation of Brain Aging. Neurotherapeutics. Vol. 16, Number 3, July 2019

21. Xia WF, Jung JU, Shun C, Xiong S, Xiong L, Shi XM, Mei L, Xiong WC.Swedish mutant APP suppresses osteoblast differentiation and causes osteoporotic deficit, which are ameliorated by $\mathrm{N}$ acetyl-L-cysteine. J Bone Miner Res. 2013 Oct;28(10):2122-35.

Publisher's Note Springer Nature remains neutral with regard to jurisdictional claims in published maps and institutional affiliations. 